\title{
A tecnologia assistiva aplicada aos casos de Transtorno do Espectro do Autismo (TEA)
}

\author{
Assistive technology applied to Autism Spectrum Disorder (ASD) \\ La tecnología asistiva aplicada a los casos de Transtorno Del Espectro Del Autismo (TEA) \\ Maria Fernanda Rocha Proença1 ${ }^{1}$, lel Marciano de Moraes Filho²*, Carla Chiste Tomazoli Santos ${ }^{1}$, \\ Tatiana Parada Romariz Rodrigues ${ }^{3}$, Débora Dadini Dantas Cangussu ${ }^{4}$, Osman Brás de Souto ${ }^{5}$.
}

\section{RESUMO}

Objetivo deste estudo fora busca refletir sobre a tecnologia assistiva e discutir sobre suas efetivas contribuições no processo da melhoria na qualidade de vida da criança com Transtorno do Espectro do Autismo (TEA). Método: Trata-se de uma revisão bibliográfica acerca da tecnologia assistiva e suas efetivas contribuições no processo de melhoria na qualidade de vida da criança com TEA. Buscou-se também analisar o Transtorno do Espectro Autista incluindo diagnóstico e a melhor maneira de lhe dar com a situação. Resultados: As discussões prevalecem no âmbito científico com a necessidade de compreensão e evolução no diagnóstico. Em relação ao diagnóstico foi levado em conta as singularidades de cada paciente a fim de intervir da melhor maneira e fora demonstrado que o manejo de transferência se destaca como uma técnica efetiva na melhora global da pessoa com TEA. Os resultados mostram uma preocupação em identificar características "autísticas" em idades cada vez mais precoces. Por outro lado, ressaltam-se impactos que uma identificação e intervenção precoce no desenvolvimento e no cotidiano dos adultos com TEA. Conclusão: Concluísse que algumas intervenções educacionais associadas a tecnologia assistiva podem contribuir para a qualidade de vida da criança com TEA. Pesquisadores destacam O método TEACCH, o ABA e SON-RISE.

Palavras-chave: Análise do Comportamento Aplicada, Autismo, Terapêutica Diagnóstico, Tecnologia Assistiva.

\begin{abstract}
Objective of this study was to reflect on the assistive technology and discuss their effective contributions in the process of improving the quality of life of children with Autism Spectrum Disorder (ASD). Method: This is a literature review about assistive technology and its effective contributions in the process of improving the quality of life of the child with ASD. It was also sought to analyze the Autism Spectrum Disorder including diagnosis and the best way to deal with the situation. Results: The discussions prevail in the scientific scope with the need for understanding and evolution in the diagnosis. Regarding the diagnosis, it was taken into account the singularities of each patient in order to intervene in the best way and it was demonstrated that transfer management stands out as an effective technique in the overall improvement of the person with ASD. The results show a concern to identify "autistic" characteristics at an earlier age. On the other hand, we highlight the impacts that an early identification and intervention in the development and in the daily life of adults with ASD. Conclusion: We conclude that some educational interventions associated with assistive technology may contribute to the quality of life of the child with ASD. Researchers highlight The TEACCH method, ABA and SON-RISE.
\end{abstract}

Keywords: Applied Behavior Analysis, Autism, Diagnostic Therapy, Assistive Technology.

\footnotetext{
${ }^{1}$ Faculdade Sena Aires (FACESA), Valparaiso de Goiás-GO.

2 Universidade Paulista (UNIP), Brasília - DF. *E-mail: ielfilho@yahoo.com.br

${ }^{3}$ Centro Universitário (UNICEPLAC), Gama - DF.

${ }^{4}$ Centro Universitário Estácio, Taguatinga- DF.

${ }^{5}$ Emil Brunner World University (EBWU), Miami - FL.
}

SUBMETIDO EM: $2 / 2019$ | ACEITO EM: $3 / 2019 \quad$ | PUBLICADO EM: 9/2019 


\section{RESUMEN}

Objetivo: El objetivo de este estudio fue buscar reflexionar sobre la tecnología asistiva y discutir sobre sus efectivas contribuciones en el proceso de la mejora en la calidad de vida del niño con Trastorno del Espectro del Autismo (TEA). Método: Se trata de una revisión bibliográfica acerca de la tecnología asistiva y sus efectivas contribuciones en el proceso de mejora en la calidad de vida del niño con TEA. Se buscó también analizar el Trastorno del Espectro Autista incluyendo diagnóstico y la mejor manera de darle con la situación. Resultados: Las discusiones prevalecen en el ámbito científico con la necesidad de comprensión y evolución en el diagnóstico. En cuanto al diagnóstico, se tuvo en cuenta las singularidades de cada paciente para intervenir de la mejor manera y se demostró que el manejo de la transferencia se destaca como una técnica efectiva en la mejora general de la persona con TEA. Los resultados muestran una preocupación en identificar características "autísticas" en edades cada vez más precoces. Por otro lado, se resaltan impactos que una identificación e intervención precoz en el desarrollo y en el cotidiano de los adultos con TEA. Conclusión: Concluir que algunas intervenciones educativas asociadas a la tecnología asistiva pueden contribuir a la calidad de vida del niño con TEA. Los investigadores destacan el método TEACCH, el ABA y SON-RISE.

Palabras clave: Análisis del comportamiento aplicado, Autismo, Terapia de diagnóstico, Tecnología assistida.

\section{INTRODUÇÃO}

Autismo é uma palavra originária do grego - autós - com o significado de "por si mesmo". O termo denomina na psiquiatria, comportamentos humanos que se centralizam em si mesmos, que se voltam ao próprio ser (CARNEIRO VB, et al.,2015). Teve surgimento pela primeira vez em 1943, vinda do médico austríaco Leo Kanner, que partiu de um estudo realizado com 11 casos diferentes, chegando ao autismo como um Distúrbio Autístico do contato afetivo, título de sua primeira publicação científica.

O Autismo traz muitos traços que afetam a afecção e evolução dos indivíduos, sendo eles o isolamento social, a falta de interação do indivíduo com o mundo exterior, a resistência a mudanças, a presença de movimentos estereotipados/repetitivos, distúrbios na linguagem/fala, a inversão pronominal, falas repetitivas, a inteligência e desenvolvimento físico, que são algumas das características mais presentes em pessoas dentro do espectro autístico (TENÓRIO MCA, 2015).

Ao longo dos últimos anos, muito se tem estudado sobre o comportamento e características da criança autista. Historicamente, trata-se de um diagnóstico recente e de um tema atual. $\mathrm{O}$ transtorno do espectro autista (TEA), o mesmo:

"[...] é caracterizado por uma série de sintomas com alterações em três áreas específicas: a socialização, a linguagem/comunicação e o comportamento. Os componentes desse trio andam "de mãos dadas" e estão intimamente relacionados" (CARNEIRO VB, et al.,2015).

Ainda se caracteriza como um conjunto de fatores específicos que afetam o desenvolvimento de diferentes formas, ocasionando geralmente comprometimento nas áreas de convivência social. Estes indivíduos apresentam padrões restritos de atividades, interesses restritos e comportamentos estereotipados. Com a longevidade dos pacientes com TEA e os avanços científicos, a forma de compreensão do mesmo tem se modificado, em níveis de eventualidades diagnósticos e a inserção de novas formas de tratamento, sendo a psicanálise um dos modelos teóricos e assistência técnica mais influentes (GONCALVES AP, et al., 2017).

O mesmo se caracteriza como um transtorno do neurodesenvolvimento caracterizado por prejuízos na comunicação, interação social e comportamental. De maneira geral, a família e os cuidadores são os primeiros a identificar alterações comportamentais na criança. As famílias que possuem crianças com o TEA apresentam um elevado nível de preocupação em relação ao bem-estar de seus filhos, ao período e as condições sob as quais permanecerão assistidos. Dessa forma, a família é considerada um agente importante no tratamento e suporte, sendo parte integrante do tratamento (MIELE FG e AMATO CAIH, 2016).

No que tange as pessoas com TEA, em 2012 houve a publicação da Lei no 12.764, considerada um marco no que tange as especificidades do educando com TEA (BRASIL, 2012). A Lei consolida a política nacional de proteção dos direitos da pessoa com Transtorno do Espectro Autista (BRASIL, 2012). 
Neste contexto um dos assuntos educacionais mais significativos da atualidade é a Tecnologia Assistiva (TA). Ela aparece juntamente com recursos múltiplos de ajuda para diminuir diferenças e potencializar a mediação no processo de aprendizagem de pessoas com deficiências. Nesta perspectiva, a TA pode contribuir no processo de Mediação da Aprendizagem da criança Autista. Os autistas apresentam déficits importantes de interação social, comunicação, linguagem e comportamento (CARNEIRO VB, et al.,2015).

Desta forma a mesma consiste em uma área do conhecimento, de característica multidisciplinar, que tem por finalidade eliminar as barreiras, incentivar à plena participação e à vida funcional para as pessoas com deficiência, incapacidades e mobilidade reduzida, objetivando autonomia e qualidade de vida (BORGES WF e MENDES EG, 2017; CARVALHO-FILHO FSS, et al.,2019).

A TA é utilizada como instrumento de acessibilidade e inclusão, e visa integrar tecnologia e inclusão em uma ferramenta capaz de atender e auxiliar alunos com necessidades educacionais especiais (TENÓRIO MCA, 2015).

A mesma e fruto do desenovelar dos avanços tecnológicos em áreas já constituídas. Desta forma e concebida em um campo do conhecimento com aspectos multidisciplinares com enfoque interdisciplinar, que englobam recursos diversos, que objetivam a melhora significativa em metodologias, estratégias, práticas que favorecem o aumento da capacidade funcional, das pessoas com deficiência, incapacidade ou mobilidade reduzida, visando a inclusão social e a melhora significativa na qualidade de vida (FERREIRA NR e RANIERI LP, 2016).

É uma disciplina de domínio de profissionais de várias áreas do conhecimento, que interagem para restaurar a função humana. Diz respeito à pesquisa, à fabricação, ao uso de equipamentos, recursos ou estratégias para potencializar as habilidades funcionais das pessoas com deficiência (MORESI EAD, et al., 2018).

O uso da TA passa por metamorfoses nas esferas culturais e pedagógica, pautadas nas novas formas de lidar com as debilidades sociais e com o conhecimento, produzido em escala geométrica e logo conduzirá a uma mudança da própria natureza da instrumentalidade, reconstruída sob o ponto de vista da arte, do respeito e do direito à convivência comunitária (CONTE E, et al.,2017).

A pesquisa feita leva em conta as Diretrizes Nacionais para Educação Especial na Educação Básica (2001), na Lei Brasileira de Inclusão da Pessoa com Deficiência, $n^{\circ}$ 13.146/15 e na lei de amparo à pessoa com autismo, lei $n^{\circ} 12.764 / 12$

O objetivo do estudo se baseia na busca da reflexão e do discursão em fontes literárias, relacionadas aos avanços das tecnologias assistivas, no processo de tratamento e melhora na qualidade de vida das crianças com TEA, baseando nos diagnósticos e logo nas melhores maneiras de gerir as situações inerentes a condição.

\section{MÉTODOS}

O presente estudo trata-se de uma revisão com caráter exploratório por meio de uma pesquisa bibliográfica, que pretende destacar a prevalência, etiologia, diagnóstico do TEA e as melhores formas de utilização da TA no processo tratamento e manejo da patologia. Para isso, foi realizada ampla pesquisa nas bases de dados MEDLINE, LILACS, BIREME, SciELO e PubMed, sendo inclusos estudos na revisão entre os anos de 2008 a 2018, totalizando 24 artigos.

A pesquisa foi realizada utilizando as bases citadas, a partir dos seguintes descritores: Transtorno do Espectro Autista; diagnóstico e Tecnologia Assistiva. Foram excluídos dissertações, teses, capítulos de livros ou trabalhos que não tratam do TEA e da tecnologia assistiva.

A amostra foi composta por 24 produções científicas para construção da pesquisa, por apresentar um objetivo mais próximo ao tema. Conforme o fluxograma abaixo: 
Figura 1 - Fluxograma para seleção da amostra que trata da etiologia, diagnóstico, tratamento do transtorno do espectro autista e a utilização da tecnologia assistiva.

\begin{tabular}{|l|l|}
\hline Amostra & Período \\
\hline 24 artigos publicados & 2008 e 2018 \\
\hline
\end{tabular}

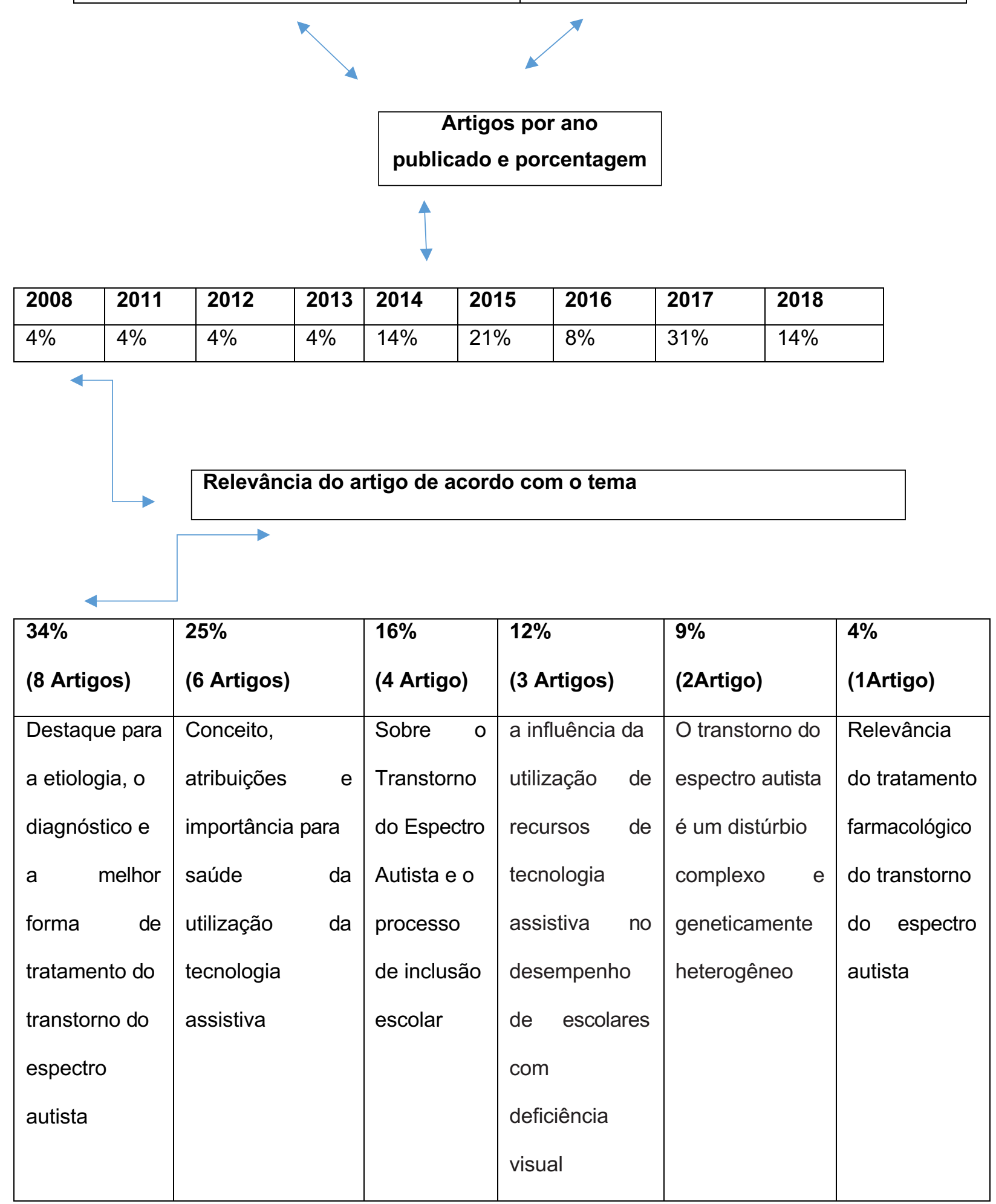

Fonte: Dados da pesquisa, 2019. 
Revista Eletrônica Acervo Saúde / Electronic Journal Collection Health | ISSN 2178-2091

Tabela 1- Relação de Artigos Incluídos na Pesquisa.

\begin{tabular}{|c|c|c|c|c|c|}
\hline Autores & Ano & Título & Objetivo & Tipo de artigo & Conclusão \\
\hline Lima CG e Menezes JP & 2008 & $\begin{array}{l}\text { Habilidade de atenção compartilhada } \\
\text { em sujeitos com } \\
\text { transtornos do espectro autístico. }\end{array}$ & $\begin{array}{l}\text { Avaliar a habilidade de atenção } \\
\text { compartilhada em sujeitos com } \\
\text { transtornos do espectro autístico } \\
\text { em diferentes contextos e com } \\
\text { diferentes } \\
\text { interlocutores }\end{array}$ & Revisão & $\begin{array}{l}\text { A avaliação da atenção compartilhada em contexto } \\
\text { de brincadeira foi eficaz e a intervenção do adulto } \\
\text { refletiu no } \\
\text { aumento destes comportamentos nas situações } \\
\text { semi-dirigidas e de imitação. }\end{array}$ \\
\hline MeccaTP, et al & 2011 & $\begin{array}{l}\text { Rastreamento de sinais e sintomas } \\
\text { de } \quad \text { transtornos } \\
\text { espectro do autismo em irmãos }\end{array}$ & 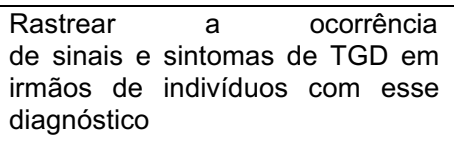 & Original & $\begin{array}{l}\text { Os presentes dados permitem recomendar às } \\
\text { equipes de saúde que avaliam crianças com } \\
\text { suspeita de TEA que também realizem, sempre } \\
\text { que possível, o rastreamento do } \\
\text { transtorno em irmãos dos probandos. }\end{array}$ \\
\hline Rocha ANDC e Deliberato D & 2012 & $\begin{array}{l}\text { Tecnologia assistiva para a criança } \\
\text { com paralisia cerebral na } \\
\text { escola: identificação } \\
\text { necessidades }\end{array}$ & $\begin{array}{l}\text { Identificar as necessidades de } \\
\text { serviços, recursos e estratégias de } \\
\text { tecnologia assistiva para o aluno } \\
\text { com paralisia cerebral na } \\
\text { escola. }\end{array}$ & Original & $\begin{array}{l}\text { O estudo identificou a necessidade de estabelecer } \\
\text { procedimentos específicos, e um } \\
\text { planejamento pedagógico organizado e a } \\
\text { participação de profissionais da saúde para o uso } \\
\text { da tecnologia assistiva na escola. }\end{array}$ \\
\hline Cruz LD, et al. & 2013 & $\begin{array}{l}\text { O fenótipo ampliado do autismo em } \\
\text { pais de indivíduos autistas: } \\
\text { uma revisão sistemática da literatura }\end{array}$ & $\begin{array}{l}\text { Realizar uma revisão sistemática } \\
\text { de estudos que } \\
\text { abordam as características } \\
\text { comportamentais relacionadas a } \\
\text { interação social, comunicação e } \\
\text { rigidez, além dos modelos } \\
\text { cognitivos } \\
\text { teoria da mente (theory of mind, } \\
\text { tom), coerência central e funções } \\
\text { executivas, em pais de indivíduos } \\
\text { autistas. }\end{array}$ & Original & $\begin{array}{l}\text { Pais de indivíduos com autismo atenuaram } \\
\text { anormalidades comportamentais na tríade de } \\
\text { sintomas centrais que caracteriza o autismo }\end{array}$ \\
\hline Carvalho KEC, et al. & 2014 & $\begin{array}{l}\text { Tradução e validação do Quebec } \\
\text { User Evaluation of Satisfaction with } \\
\text { Assistive Technology (QUEST 2.0) } \\
\text { para o idioma português do } \\
\text { Brasil }\end{array}$ & $\begin{array}{l}\text { Traduzir e validar o Quebec User } \\
\text { Evaluation of Satisfaction with } \\
\text { Assistive } \\
\text { (QUEST 2.0) para o idioma } \\
\text { português do Brasil. }\end{array}$ & Original & $\begin{array}{l}\text { Foram obtidos dados de } 121 \text { usuários dos } \\
\text { dispositivos mencionados. O estudo demonstrou } \\
\text { um IVC de } 91,66 \% \text { e uma análise de fatores } \\
\text { satisfatória com referência à estrutura } \\
\text { bidimensional do instrumento, o que assegurou a } \\
\text { representatividade dos itens }\end{array}$ \\
\hline Rabello S, et al. & 2014 & $\begin{array}{l}\text { A influência de recursos de } \\
\text { tecnologia assistiva sobre } \\
\text { a performance em atividades de } \\
\text { deficientes visuais }\end{array}$ & $\begin{array}{l}\text { Verificar a influência da utilização } \\
\text { de recursos de tecnologia assistiva } \\
\text { no desempenho de escolares com } \\
\text { deficiência visual } \\
\text { em atividades em sala de recursos }\end{array}$ & Original & $\begin{array}{l}\text { Constatou-se o melhor desempenho das } \\
\text { habilidades de leitura, após o uso de recursos de } \\
\text { tecnologia assistiva, o que facilitou a realização } \\
\text { de atividades acadêmicas emigualdade } \\
\text { aos colegas da sala. }\end{array}$ \\
\hline Tamanaha AC, et al. & 2014 & $\begin{array}{l}\text { Trajetória de aquisição } \mathrm{e} \\
\text { desenvolvimento de fala de crianças } \\
\text { autistas com e sem história de } \\
\text { regressão autística }\end{array}$ & $\begin{array}{l}\text { Comparar a trajetória de aquisição } \\
\text { e desenvolvimento de fala de } \\
\text { crianças autistas com e sem } \\
\text { história } \\
\text { de regressão autística. }\end{array}$ & Original & 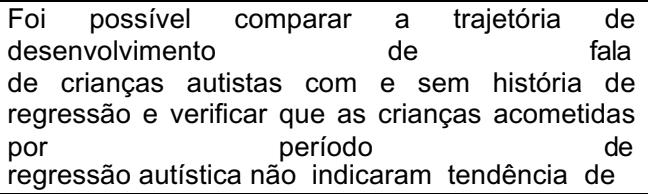 \\
\hline
\end{tabular}

\footnotetext{
REAS/EJCH | Vol.Sup.31 | e541 | DOI: https://doi.org/10.25248/reas.e541.2019 Página 5 de 13
} 
Revista Eletrônica Acervo Saúde / Electronic Journal Collection Health | ISSN 2178-2091

\begin{tabular}{|c|c|c|c|c|c|}
\hline & & & & & $\begin{array}{l}\text { desfecho clínico mais positivo em relação à } \\
\text { produção de fala. }\end{array}$ \\
\hline Schwartzman JS, et al. & 2015 & $\begin{array}{l}\text { Rastreamento do olhar para } \\
\text { estímulos sociais em pacientes com } \\
\text { síndrome de Rett e } \\
\text { transtornos do espectro do autismo: } \\
\text { estudo piloto }\end{array}$ & $\begin{array}{l}\text { Comparar a fixação visual em } \\
\text { estímulos sociais em pacientes } \\
\text { com síndrome de Rett (SR) e com } \\
\text { transtornos do espectro do autismo } \\
\text { (TEA) }\end{array}$ & Original & $\begin{array}{l}\text { A fixação visual em estímulos sociais parece ser } \\
\text { mais um dos endofenótipos que esclarecem as } \\
\text { diferenças entre SR e TEA. }\end{array}$ \\
\hline Almeida MC, et al. & 2015 & 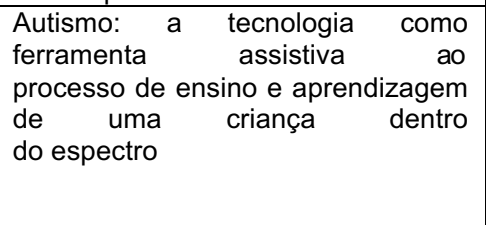 & $\begin{array}{l}\text { Analisar como o ipad pode ser } \\
\text { utilizado } \text { como r uma } \\
\text { ferramenta nos processos de } \\
\text { ensino e aprendizagem de uma } \\
\text { criança } \\
\text { autismo. }\end{array}$ & Original & $\begin{array}{l}\text { Deste modo, acredita-se que o uso da tecnologia } \\
\text { traz ferramentas assistivas que } \\
\text { podem ser eficazes em prol do desenvolvimento de } \\
\text { crianças com autismo inseridas em classe regular, } \\
\text { sendo essa uma forma de facilitar o processo de } \\
\text { ensino a e a prendizagem } \\
\text { desses alunos }\end{array}$ \\
\hline Carneiro VB, et al. & 2015 & $\begin{array}{l}\text { A tecnologia assistiva no processo } \\
\text { de } \quad \text { mediação } \\
\text { aprendizagem do aluno autista }\end{array}$ & \begin{tabular}{llr}
\multicolumn{3}{l}{ Refletir sobre a TA e discutir sobre } \\
as suas efetivas contribuições no \\
processo & mediático ro na \\
aprendizagem & do aluno com \\
necessidades & \multicolumn{2}{c}{ educacionais } \\
especiais. & & \\
\end{tabular} & Original & $\begin{array}{l}\text { A tecnologia assistiva tem como aliada a } \\
\text { tecnologia digital e ambas mostram um leque de } \\
\text { possibilidades de práticas educativas mediáticas } \\
\text { bem contextualizadas. }\end{array}$ \\
\hline Pereira ACS, et al. & 2015 & $\begin{array}{l}\text { Transtorno do Espectro Autista } \\
\text { (TEA): } \\
\text { definição, características e } \\
\text { atendimento } \\
\text { educacional }\end{array}$ & $\begin{array}{l}\text { Apresentar uma visão geral acerca } \\
\text { das características da criança com } \\
\text { Transtorno } \\
\text { do Espectro do Autismo (TEA) e o } \\
\text { atendimento educacional a ser } \\
\text { oferecido }\end{array}$ & Original & $\begin{array}{l}\text { O atendimento educacional especializado e o } \\
\text { trabalho colaborativo entre professor de ensino } \\
\text { comum e professor de educação especial são } \\
\text { ferramentas fundamentais para o atendimento } \\
\text { adequado e aprendizagem efetiva dos } \\
\text { alunos com TEA. }\end{array}$ \\
\hline Zauza CMF, et al. & 2015 & $\begin{array}{l}\text { O processo de inclusão de } \\
\text { portadores do transtorno do espectro } \\
\text { autista }\end{array}$ & $\begin{array}{llr}\text { Classificar } & \text { definições } & \text { do } \\
\text { transtorno, } & \text { estratégias } & \text { de } \\
\text { assistência e intervenção } & \text { e } \\
\text { aspectos relacionados à inclusão } \\
\text { no ensino regular. }\end{array}$ & Original & $\begin{array}{l}\text { O Diagnóstico do TEA tem se consolidado durante os } \\
2 \text { anos de idade o tratamento na maioria dos casos e } \\
\text { multiprofissional embora não haja uma concordância } \\
\text { específica no tipo de tratamento. }\end{array}$ \\
\hline Calheiros DS e Mendes EG & 2016 & $\begin{array}{lr}\text { Consultoria } & \text { colaborativa } \\
\text { a distância em } & \text { tecnologia assistiva } \\
\text { para professores } & \end{array}$ & $\begin{array}{l}\text { Avaliar um serviço de consultoria } \\
\text { colaborativa } \\
\text { - scc - a distância em tecnologia } \\
\text { assistiva - ta - para professores, } \\
\text { utilizando } \\
\text { abordagem qualitativa do tipo } \\
\text { exploratório. }\end{array}$ & Original & 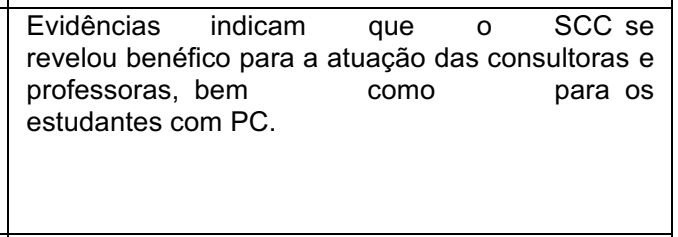 \\
\hline Miele FG e Amato CALH & 2016 & $\begin{array}{l}\text { Transtono do espectro autista: } \\
\text { qualidade de vida e estresse } \\
\text { em cuidadores e/ou familiares - } \\
\text { revisão de literatura }\end{array}$ & $\begin{array}{l}\text { Analisar artigos relacionados ao } \\
\text { estresse e qualidade de vida de } \\
\text { familiares e/ou cuidadores de } \\
\text { crianças com TEA. }\end{array}$ & Original & $\begin{array}{lrrr}\text { O resultado mostrou que os problemas de } & \text { que } \\
\text { comunicação, } & \text { comportamento } & \text { e } \\
\text { interesse apresentados pela criança impactaram } \\
\text { na qualidade de vida materna. } & \text { Foram } \\
\text { encontradas } & \text { diferenças entre meninos e } \\
\text { meninas } & & & \\
\end{array}$ \\
\hline
\end{tabular}

\footnotetext{
REAS/EJCH | Vol.Sup.31 | e541 | DOI: https://doi.org/10.25248/reas.e541.2019 Página 6 de 13
} 
Revista Eletrônica Acervo Saúde / Electronic Journal Collection Health | ISSN 2178-2091

\begin{tabular}{|c|c|c|c|c|c|}
\hline Borges WF e Tartuci D & 2017 & $\begin{array}{l}\text { Tecnologia assistiva: concepções de } \\
\text { professores e as problematizações } \\
\text { geradas pela imprecisão conceitual }\end{array}$ & 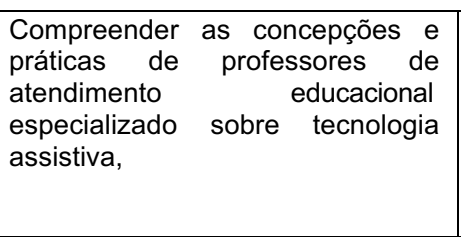 & Original & $\begin{array}{l}\text { Pela observação dos aspectos analisados neste } \\
\text { artigo, o que evidenciamos, em geral, é que as } \\
\text { professoras têm buscado a ludicidade, materiais } \\
\text { concretos, diferentes suportes textuais e } \\
\text { atividades pedagógicas com auxilio das tics como } \\
\text { estratégias para desenvolver habilidades dos seus } \\
\text { alunos. }\end{array}$ \\
\hline Conte $\mathrm{E}$, et al. & 2017 & $\begin{array}{lcr}\text { Tecnologia } & \text { assistiva, } & \text { direitos } \\
\text { humanos } & \text { e } & \text { educação } \\
\text { inclusiva: uma nova sensibilidade }\end{array}$ & $\begin{array}{l}\text { Discutir sobre o dispositivo } \\
\text { pedagógico da tecnologia assistiva } \\
\text { no processo de de } \\
\text { reconhecimento mútuo, de } \\
\text { interação social e de construção da } \\
\text { aprendizagem, } \\
\text { para promover a igualdade de } \\
\text { direitos e o exercício da cidadania. }\end{array}$ & Original & $\begin{array}{l}\text { O uso da TA por si só não garante novas formas de } \\
\text { aprender com } \\
\begin{array}{llll}\text { as diferenças e pode gerar a } \\
\text { valorização } & \text { apenas } & \text { da } & \text { repetição }\end{array}\end{array}$ \\
\hline Fachinetti TA, et al. & 2017 & $\begin{array}{l}\text { Processo de construção de recurso } \\
\text { de tecnologia assistiva para aluno } \\
\text { com paralisia cerebral em sala de } \\
\text { recursos multifuncionais1 }\end{array}$ & $\begin{array}{l}\text { Implementar e avaliar um recurso } \\
\text { de tecnologia assistiva para um } \\
\text { aluno paralisia } \\
\text { cerebral de forma colaborativa com } \\
\text { a professora da sala de recursos } \\
\text { multifuncionais, por meio do } \\
\text { fluxograma apresentado na } \\
\text { literatura }\end{array}$ & Original & $\begin{array}{l}\text { Com os resultados evidenciados neste estudo, } \\
\text { percebeu-se que a utilização da } \\
\text { tecnologia assistiva não fazia parte do cotidiano } \\
\text { dos atendimentos realizados na sala de recursos } \\
\text { multifuncionais pela professora participante. }\end{array}$ \\
\hline Gonçalves AP, et al. & 2017 & $\begin{array}{l}\text { Transtornos do espectro do autismo } \\
\text { e psicanálise: revisitando a literatura }\end{array}$ & $\begin{array}{l}\text { Analisar as relações entre autismo } \\
\text { e psicanálise em produções } \\
\text { bibliográficas brasileiras do } \\
\text { período } \\
\text { de } 2009 \text { a } 2014 \text { e analisar sistema } \\
\text { ticamente as publicações sobre as } \\
\text { relações entre os TEA e a } \\
\text { psicanálise }\end{array}$ & Revisão & $\begin{array}{l}\text { Os resultados obtidos indicaram que o maior } \\
\text { volume de obras trata a etiologia como multicausal, } \\
\text { destacando aspectos genéticos, biológicos, } \\
\text { psicogênicos e relacionais. Sobre o diagnóstico, } \\
\text { foram } \\
\text { ressaltadas a singularidade e a subjetividade de } \\
\text { cada sujeito e com relação ao tratamento } \\
\text { enfatizou-se o manejo da transferência como uma } \\
\text { técnica } \\
\text { apropriada. }\end{array}$ \\
\hline Monteiro AF, et al. & 2017 & $\begin{array}{lcc}\text { Considerações } & \text { sobre } & \text { critérios } \\
\text { diagnósticos } & \text { de } & \text { transtorno } \\
\text { do espectro autista, e } & \text { e suas } \\
\text { implicações no campo científico }\end{array}$ & $\begin{array}{l}\text { Revisão bibliográfica acerca do } \\
\text { diagnóstico de TEA }\end{array}$ & Revisão & $\begin{array}{l}\text { Os resultados encontrados mostram uma } \\
\text { preocupação em identificar características } \\
\text { artísticas em idades cada vez mais precoces. } \\
\text { Essa identificação permite trabalhar } \\
\text { atenuar possíveis impactos que comportamento c } \\
\text { aracterístico do TEA possa causar na vida dessas } \\
\text { pessoas com o transtorno. }\end{array}$ \\
\hline
\end{tabular}

\footnotetext{
REAS/EJCH | Vol.Sup.31 | e541 | DOI: https://doi.org/10.25248/reas.e541.2019 Página 7 de 13
} 
Revista Eletrônica Acervo Saúde / Electronic Journal Collection Health | ISSN 2178-2091

\begin{tabular}{|c|c|c|c|c|c|}
\hline Kelly RS, et al. & 2017 & $\begin{array}{l}\text { Transtorno do espectro do autismo } \\
\text { (TEA): do reconhecimento à } \\
\text { inclusão no âmbito educacional }\end{array}$ & 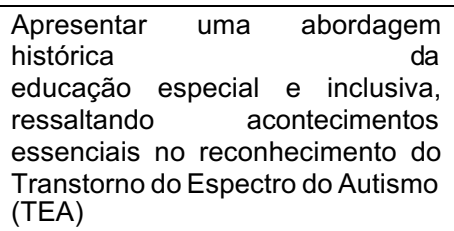 & Revisão & $\begin{array}{l}\text { Reconhecimento do TEA é o início para que sejam } \\
\text { efetivadas ações significativas, havendo uma } \\
\text { ligação dialógica da família com os profissionais da } \\
\text { saúde e da educação para inclusão do sujeito com } \\
\text { autismo no âmbito educacional }\end{array}$ \\
\hline $\begin{array}{lllll}\text { Borges } & \text { WF } & \text { e } & \text { Mendes } & \text { EG }\end{array}$ & 2018 & $\begin{array}{l}\text { Usabilidade de aplicativos de } \\
\text { tecnologia assistiva por pessoas } \\
\text { com baixa visão }\end{array}$ & $\begin{array}{l}\text { Identificar e caracterizar funcional } \\
\text { mente, a partir do ponto de vista } \\
\text { dos usuários, aplicativos de } \\
\text { smartphones e/ou tablets que } \\
\text { assumem de função } \\
\text { recursos de TA e vêm sendo de } \\
\text { utilizados por pessoas com baixa } \\
\text { visão }\end{array}$ & $\begin{array}{l}\text { Relato } \\
\text { pesquisa }\end{array}$ & $\begin{array}{l}\text { Foi possível identificar o potencial desses } \\
\text { aplicativos na solução de dificuldades enfrentadas } \\
\text { por pessoas com baixa visão. } \\
\text { Desse modo, sugere-se mais pesquisas, } \\
\text { investimentos, divulgação e programas de ensino } \\
\text { para aumentar o seu uso. }\end{array}$ \\
\hline Moresi EAD, et al. & 2018 & Tecnologia assistiva e autismo & $\begin{array}{l}\text { Apresentar } \quad \text { o emprego de } \\
\text { tecnologia } \\
\text { desenvolvida para dispositivo } \\
\text { móvel, para ajudar autistas em } \\
\text { suas atividades diárias. }\end{array}$ & Revisão & $\begin{array}{l}\text { A utilização da metodologia CBL (Challenge Based } \\
\text { Learning) foi um fator muito importante para } \\
\text { solucionar o problema de forma eficiente }\end{array}$ \\
\hline Seabra Junior MO e Lacerda LCZ & 2018 & $\begin{array}{l}\text { Atendimento educacional } \\
\text { especializado: planejamento e uso } \\
\text { do recurso pedagógico }\end{array}$ & $\begin{array}{l}\text { Analisar as contingências a que o } \\
\text { professor do atendimento } \\
\text { educacional especializado está } \\
\text { submetido ao realizar o } \\
\text { desenvolvimento e aplicação dos } \\
\text { recursos pedagógicos necessários } \\
\text { aos estudantes da sala de recurso } \\
\text { multifuncional }\end{array}$ & Revisão & $\begin{array}{l}\text { Quanto ao desenvolvimento e aplicação dos } \\
\text { recursos de TA em ambos os ambientes (SRM e } \\
\text { sala comum), observou-se por meio da } \\
\text { análise que a somatória do uso de um fluxograma } \\
\text { de avaliação de TA adicionada à práxis dos } \\
\text { professores especialistas proporciona novos } \\
\text { indicativos na conjuntura } \\
\text { de ações do professor diante das diferentes } \\
\text { realidades e necessidades de seus estudantes. }\end{array}$ \\
\hline Griesi-Oliveira K e Sertié AL & 2017 & $\begin{array}{l}\text { Transtornos do espectro autista: } \\
\text { um guia atualizado para } \\
\text { aconselhamento genético }\end{array}$ & $\begin{array}{l}\text { Apresentar uma breve discussão } \\
\text { sobre a visão atual da arquitetura } \\
\text { genética dos transtornos do } \\
\text { espectro autista, }\end{array}$ & Revisão & $\begin{array}{l}\text { A rápida evolução do conhecimento proporcionada } \\
\text { pelas pesquisas }\end{array} \begin{array}{rr}\text { genéticas } \\
\text { relacionadas ao autismo certamente } \\
\text { contribuirá para o desenvolvimento de técnicas } \\
\text { diagnósticas } \quad \text { mais } & \text { precisas } \\
\text { e, possivelmente, para terapias baseadas em } \\
\text { evidências genéticas, tornando a investigação da } \\
\text { etiologia genética do TEA em crianças ainda } \\
\text { mais importante. }\end{array}$ \\
\hline
\end{tabular}

Fonte: Dados da pesquisa, 2019.

REAS/EJCH | Vol.Sup.31 | e541 | DOI: https://doi.org/10.25248/reas.e541.2019 Página 8 de 13 


\section{RESULTADOS E DISCUSSÃO}

A pesquisa feita ressalta que a etiologia e o diagnóstico e a melhor forma de tratamento para o TEA e foram descritas em 08 artigos (34\%). Todavia, destaca-se também o conceito, atribuições e importância para saúde da utilização da TA, em 06 artigos (25\%). Vale ressaltar que quatro artigos enfatizam (16\%) sobre o TEA e o processo de inclusão escolar que foi destacado em 03 (12\%), sumarizando a respeito da influência e da utilização de recursos de TA no desempenho do escolar com deficiência visual. Foi identificado também 02 artigos (9\%) que relatam sobre o TEA e os distúrbios complexos e geneticamente heterogêneo e 01 artigo (4\%) que identificou a relevância do tratamento farmacológico no TEA.

No espaço de tempo delimitado para a realização deste estudo (2008-2018) foram encontradas e analisadas vinte e quatro publicações. Nos anos de 2008, 2011, 2012 e 2013 teve um artigo respectivamente perfazendo (4\%) que foi utilizado na revisão bibliográfica. Em 2014 três artigos totalizando (14\%), em 2015 cinco artigos (21\%), no ano de 2016 teve dois artigos (8\%), em 2017 teve o maior número de publicações 7 (31\%), por fim no ano de 2018 destaca-se a utilização de 03 artigos (14\%) que contribuíram para a pesquisa sobre a TA e o TEA.

Dentre as publicações analisadas, três enfatizam que o TEA se trata de um distúrbio no desenvolvimento humano, caracterizado por vários prejuízos aos seus portadores no que se refere à interação social, à comunicação verbal e não verbal (SCHWARTZMAN JS, et al., 2015; SANTOS RK, 2017; MORESI EAD, et al., 2018).

Outros quatro artigos elaboram reflexões críticas às características das crianças com TEA. Elas apresentam uma ausência de habilidade para o estabelecimento de jogos variados e espontâneos de imaginação ou de imitação, apropriados aos níveis de desenvolvimento humano individual, assim como uma tendência para o estabelecimento de comportamentos rígidos e repetitivos, e interesse por rotinas ou rituais não funcionais (ROCHA ANDC E DELIBERATO D,2012;MONTEIRO AF, et al.,2017; Seabra Junior MO e Lacerda LCZ, 2018; BORGES WF, MENDES EG, 2018).

Do total de vinte e quatro artigos selecionados, constatou-se que oito enfatizaram a importância de ofertar serviços de atendimento atentando para o fato de que não há um manual a ser seguido, é fundamental aprender a lidar com as particularidades de cada sujeito para, assim, obter maior êxito nessas intervenções(MENEZES CGL e PERISSINOTO J , 2008; MECCA TP, et al., 2011; CRUZ LP, et al.,2013; RABELLO S, et al., 2014;PEREIRA ACS et al., 2015; MIELE FG e AMATO CAIH, 2016; MONTEIRO AF, et al.,2017; BORGES WF, MENDES EG, 2018).

Em 05 publicações fora considerado que os métodos de acolhimento, a espontaneidade e a criatividade do analista são estratégias que possibilitam de maneira efetiva a entrada no campo psíquico do paciente. As mesmas são consideradas intervenções para o TEA, pois destacam as interpretações de gestos representativos ou automatismos (SCHWARTZMAN JS, et al., 2015; SANTOS RK, 2017; MONTEIRO AF, et al.,2017; SEABRA JUNIOR MO E LACERDA LCZ 2018; MORESI EAD, et al., 2018).

Quatro publicações versam sobre a importância da percepção das características da pessoa com autismo e não podem ser consideradas como motivos de desistência nos aspectos pessoal, educacional e profissional, é um desafio, e os primeiros passos a serem tomados é conhecer, acompanhar e buscar cada vez mais por melhores condições para o desenvolvimento cognitivo, afetivo e social (SCHWARTZMAN JS, et al., 2015; PEREIRA ACS, et al., 2015; MONTEIRO AF, et al., 2017; SANTOS RK, 2017).

O estudo de Moresi EAD et al. (2008) destaca que os TEA se referem ao desenvolvimento neurológico, caracterizados por deficiências na interação social, na comunicação (ou seja, linguagem verbal e não verbal), por interesses restritos e comportamentos repetitivos. No entanto, a aplicação de robôs, como ferramenta terapêutica, mostrou resultados promissores, particularmente por causa da capacidade de melhorar o engajamento social ao provocar comportamentos sociais apropriados em crianças com tal espectro.

Três publicações reconhecem que o TEA é um distúrbio complexo e geneticamente heterogêneo, o que sempre dificultou a identificação de sua etiologia em cada paciente em particular e por consequência, o 
aconselhamento genético das famílias. Assim, em vista do conhecimento atual sobre a arquitetura genética do TEA, que tem tornado o aconselhamento genético cada vez mais preciso, e dos potenciais benefícios que a investigação etiológica pode trazer aos pacientes e familiares, tornam-se cada vez mais importantes os testes genéticos moleculares (MECCA TP, et al., 2011;MONTEIRO AF, et al.,2017; GRIESIOLIVEIRA K e SERTIE AL, 2018).

Cinco artigos destacaram que a necessidade de estabelecer procedimentos específicos, um planejamento pedagógico organizado e a participação de profissionais da saúde para o uso da TA na escola, possibilita um melhor desempenho das habilidades de leitura, após o uso de recursos de TA, o que facilitou a realização de atividades acadêmicas em igualdade aos colegas da sala. Todavia, o uso da TA por si só não garante novas formas de aprender com as diferenças e pode gerar a valorização apenas da repetição (ROCHA ANDC E DELIBERATO D,2012; RABELLO S, et al.2014; FACHINETTI TA, et al.,2017; CONTE E, et al.,2017; MORESI EAD, et al.,2018).

Um artigo destacou sobre o diagnóstico, e sugere que a singularidade e a subjetividade de cada sujeito têm fundamental importância para o tratamento do TEA e também que o manejo da transferência dos cuidadores pode ser uma técnica apropriada (GONCALVES AP, et al, 2017).

Fora possível identificar no estudo de CARNEIRO VB ET AL. (2015) que alguns grupos de estudo em universidades brasileiras estão desenvolvendo a TA com a criação de softwares específicos para mediar e facilitar a oralidade de crianças com TEA.

Para melhor compreensão os resultados foram divididos em três vertentes: 1- Etiologia, diagnóstico do TEA; 2- Fatores associados aos casos de TEA e a melhor maneira de tratamento e 3- A Tecnologia Assistiva ajudando na qualidade de vida do autista e sua família, pois para a aplicação de tecnologias assistivas e preciso compreender o contexto vivenciado pela pessoa coma TEA.

\section{Etiologia, diagnóstico do TEA}

Em 2013 ocorreu a publicação do DMS 5, em que os transtornos abordados foram: Transtorno Autista, Transtorno Desintegrativo da Infância, Transtorno de Asperger e Transtorno Invasivo do Desenvolvimento Sem Outra Especificação. Tal publicação trouxe inúmeras mudanças nos critérios abordados nos diagnósticos de autismo, em que se adotou o termo TEA, somente a Síndrome de Rett foi excluída da categoria, e foi considerada uma doença distinta (ZAUZA CMF, et al., 2015).

O autismo infantil é uma síndrome comportamental caracterizada por uma tríade de sintomas: déficits nas interações sociais, déficits na comunicação verbal e não verbal e inflexível, comportamentos e interesses restritos e estereotipados, intensidade destes sintomas pode variar de leve em que indivíduo tem um alto potencial intelectual, a grave, em que existe retardo mental acentuado levando até a falta da oralidade (CRUZ LP, et al.,2013).

A palavra espectro abrange as variantes de intensidade de sintomas e situações que a pessoa autista pode apresentar, numa nuance que vai da mais severa, definindo o autismo de baixo funcionamento, até a mais leve, determinando o autismo de alto funcionamento (CARNEIRO VB, et al.,2015;TAMANAHA AC, et al.,2014).

A maior quantidade de identificação desta doença nos dias de hoje, deve-se, provavelmente, ao fato que os estudos ampliaram o conhecimento sobre o TEA e seu diagnóstico, e estima-se que de cada 100 pessoas uma é afetada pelo transtorno, o que mostra um aumento considerável em relação a dados referenciados há algumas décadas. Sobre as causas do autismo infantil, ainda inconclusivas hoje em dia, sabe-se que, além de um grave transtorno do neurodesenvolvimento de etiologia (s) desconhecida (s), há evidências genéticas relacionadas (BORGES WF, MENDES EG, 2018).

Contudo, é perceptível que o diagnóstico do TEA vem se consolidando para crianças até dois anos de idade, que apresentam bom desempenho ao tratamento. Além disso, o tratamento é altamente eficaz, embora não tenha uma relação específica ao tipo de tratamento. 
Atualmente, o autismo está classificado na categoria dos transtornos globais do desenvolvimento (TGD) ou transtornos invasivos do desenvolvimento em manuais de classificação, tais como o Diagnostic and Statistical Manual of Mental Disorders, 4th edition, Text Revision (DSM-IV-TR) e a Classificação Estatística de Doenças e Problemas Relacionados à Saúde - 10a Revisão (CID-10), respectivamente (MECCA TP, et al.,2011).

Os resultados apontaram que os pais de indivíduos autistas possuem déficits na interação social, na linguagem pragmática e traços de rigidez (CRUZ LP, et al., 2013).

Convém destacar que a aplicação de protocolos amplos de maneira sistemática no estudo de casos de indivíduos com TEA deve prever a investigação genealógica e a aplicação de questionários de rastreamento de sinais e sintomas em consanguíneos dos probandos, principalmente em irmãos (MECCA TP, et al., 2011).

Dessa forma, algumas crianças estabelecem mudanças de frequência em escolas, algumas frequentaram uma escola e logo mudam para outras devido a incapacidade formativa dos educadores e aporte da instituição de ensino.

Os pais observam que há diversificação da maneira de ação entre as escolas, algumas possuem orientações especificas e professores capacitados. Enquanto em algumas escolas é defasado as condições adequadas de atendimento às crianças com TEA. Levando em consideração o relacionamento entre as crianças, é satisfatório na percepção dos pais, independente da escola frequentada, mas existem diversidades, em que algumas têm maiores dificuldades de interação social e comunicação (TAMANAHA AC, et al., 2014).

\section{Fatores associados aos casos de TEA e a melhor maneira de tratamento}

Enquanto alguns pesquisadores desenvolvem uma linha de investigação pautada em olhares psicopedagógicos, defendendo o uso de fármacos, como a oxitocina, para atenuar os sintomas de sociabilização, tendo em vista que as pesquisas sobre o transtorno estariam avançando em direção a uma causa genética mais claramente definida. No caso, encorajar-se-ia, assim, a indústria farmacêutica a entrar também nesse setor. Ora, um dos maiores obstáculos no estudo clínico da oxitocina se dá devido à complexidade para se medir alterações no chamado comportamento social, que, não sem importância, é um dos sintomas nucleares mais complicados no TEA (EISENSTEIN M,2012; MONTEIRO AF, et al.,2017).

A demanda de cuidados aos quais uma criança com TEA necessita e as mudanças na rotina dos familiares tais como, hábitos, gastos financeiros, relações sociais e profissionais, podem ser percebidas como um evento estressor para os membros da família, cuidadores e pessoas mais próximas, podendo acarretar uma sobrecarga física e mental (MIELE FG e AMATO CAIH, 2016).

As crianças que portam TEA possuem características específicas tais como, dificuldade/falta de comunicação, pouco/nenhum contato visual, apáticos e retraídos (ZAUZA CMF, et al., 2015). Existem técnicas que auxiliam no tratamento dessas características, tais como a musicoterapia. O uso dessa terapia em crianças com TEA se realiza com o desenvolvimento de aspecto sócio emocionais, incentivando o comportamento verbal e não verbal, diminui assim os problemas resultantes de percepção e função motora. Sendo assim, tornando-as melhores no desenvolvimento e promovendo satisfação emocional.

Algumas intervenções psicoeducacionais, associada a tecnologia assistiva podem contribuir para a qualidade de vida da criança com TEA. Pesquisadores destacam O método TEACCH, o ABA e SON-RISE.

O método TEACCH, se baseia em uma avaliação denominada PEP-R (Perfil Psicoeducacional Revisado), para avaliar a criança com TEA, identificando os seus pontos fortes e de maior interesse, e logo as suas dificuldades de maneira operacional. A fim de planejar um programa individualizado, que organize o espaço físico, desenvolva horários, e sistemas de trabalho, esclarecendo e explicitando as esperanças e utilizando métodos visuais, pontuando de maneira inerente o ambiente de convivência social e de aprendizagem, através da ação clara, objetivando proporcionar uma estrutura para todo o ensino. Desta forma permitindo ao 
aluno uma independência, em grande parte do seu tempo, utilizando o educador apenas para o aprendizado de novas atividades (PEREIRA ACS, et al., 2015).

O método ABA se concentra na medição confiável e avaliação objetiva do comportamento observável possibilitando a melhora do comportamento, o ensino de novas habilidades, a manutenção de comportamentos, a capacitação para Generalizar ou transferir o comportamento de uma situação ou resposta a outra para a complementação de tarefas e também avalia para restringir ou condicionar situações estreitas, sob o qual ocorrem comportamentos que interferem no desenovelar do desempenho através da explicação da associação do ambiente no comportamento da pessoa com TEA (PEREIRA ACS ,et al., 2015).

O método SON-RISE, se baseia na aproximação e demonstração de aceitação, através dos movimentos estereotipados no momento em que a criança com TEA os realiza, a repetição dos mesmos pelos pais poderá demostrar aceitação e também é um momento oportuno a identificar as preferências sensórias para o planejamento de atividades, após a aceitação do método pela criança com TEA, assim poderá se propor novas atividades (PEREIRA ACS, et al., 2015).

\section{A Tecnologia Assistiva ajudando na qualidade de vida do autista e sua família}

Os estudos sobre TA enfatizaram a necessidade de inserir recursos, serviços e estratégias na educação especial e inclusiva para colaborar com o processo de aprendizagem de alunos com deficiências. A literatura descreveu que a primeira etapa para a implementação da TA na escola deve permitir entender a situação que envolve o aluno a fim de ampliar a sua participação no processo de ensino e aprendizagem (ROCHA ANDC E DELIBERATO D, 2012).

Documentos internacionais e nacionais têm procurado definir a TA e garantir políticas públicas que favoreçam o seu uso. Na literatura internacional as definições de TA enfatizaram o uso de recursos, estratégias e serviços aplicados para atenuar os problemas funcionais encontrados pelos indivíduos com deficiências e proporcionar ou ampliar suas habilidades e consequentemente, promover independência, qualidade de vida e inclusão (ROCHA ANDC E DELIBERATO D, 2012; TENÓRIO MCA, et al.,2015).

$O$ que se entende na contemporaneidade sobre TEA, consiste num acervo de componentes específicos percebidos nos sujeitos geralmente até os três anos de idade, pontuando entre esses um comportamento alheio caracterizado pela ineficiência social e isolamento, provocados pela aversão aos estímulos externos, que podem passar despercebidos aos olhos nus, além das particularidades no estabelecimento de vínculos afetivos e das amostras de comunicação verbal e não verbal (GONCALVES AP, et al, 2017; Seabra Junior MO e Lacerda LCZ ,2018).

Os resultados demonstraram que após entender a situação do aluno com deficiência no contexto escolar foi possível estabelecer as suas habilidades e necessidades para indicar os recursos de TA adequados ao planejamento do professor e propiciar a aprendizagem da criança com deficiência. O estudo identificou a necessidade de estabelecer procedimentos específicos, um planejamento pedagógico organizado e a participação de profissionais da saúde para o uso da TA (ROCHA ANDC E DELIBERATO D,2012; SCHWARTZMAN JS, et al., 2015).

Constatou-se o melhor desempenho das habilidades de leitura, após o uso de recursos de TA, o que facilitou a realização de atividades acadêmicas em igualdade aos colegas da sala (RABELLO S, etal.,2014).

Sendo assim, a TA atua como um importante instrumento que auxilia a promoção e ampliação de habilidades funcionais, deficitárias ou permitirá a realização da função desejada, que se encontra atalhada por circunstância de deficiência ou ocasionada pelo processo de senescência (CARVALHO KEC, et al.,2014; MENEZES CGL e PERISSINOTO J, 2008;CARVALHO FILHO et al., 2018; CARVALHO FILHO et al., 2018).

\section{CONCLUSÃO}

O diagnóstico do TEA é clínico e baseia-se na presença de determinados padrões de comportamento: déficits clinicamente significativos e persistentes na comunicação e interações sociais, manifestadas de todas as maneiras seguintes; déficits expressivos na comunicação não verbal e verbal usadas para interação social; 
e padrões restritos e repetitivos de comportamento, interesses e atividades. Visando o levantamento de diagnósticos, foram ressaltadas as necessidades individuas de cada sujeito, e relacionado ao tratamento concluísse o manejo da transferência como uma técnica adequada. Sendo assim, é necessário a incorporação de estudos no contexto mundial, a fim de integrar a sociedade para colaborar no auxílio do desenvolvimento do tratamento para o TEA.

\section{REFERÊNCIAS}

1. BORGES WF, MENDES EG. Usabilidade de Aplicativos de Tecnologia Assistiva por Pessoas com Baixa Visão. Revista brasileira de educação espec. 2018;24(4):483-500.

2. BORGES WF, TARTUCI D. Tecnologia Assistiva: Concepções de Professores e as Problematizações Geradas pela Imprecisão Conceitual1. Rev. bras. educ. espec. 2017;23(1):81-96.

3. CALHEIROS DS, MENDES EG. Consultoria colaborativa a distância em tecnologia assistiva para professores. Caderno de Pesquisa. 2016; 46(162):1100-1123.

4. CARNEIRO VB. A tecnologia assistiva no processo de aprendizagem do aluno autista. EDUCERE. PUCPR. 2015.2 Disponível https://educere.bruc.com.br/arquivo/pdf2015/16117 7472.pdf . Acesso em: 24 de ago.de 2019.

5. CARVALHO KEC, et al. Tradução e validação do Quebec User Evaluation of Satisfaction with Assistive Technology (QUEST 2.0) para o idioma português do Brasil. Rev. Bras. Reumatol. 2014; 54(4):260-267.

6. CONTE E, et al. Tecnologia assistiva, direitos humanos e educação inclusiva: uma nova sensibilidade. Educ. rev. 2017; 33:e163600.

7. CRUZ LP, et al. The broad autism phenotype in parents of individuals with autism: a systematic review of the literature. Trends Psychiatry Psychother. 2013;35(4):252-263.

8. EISENSTEIN M. Treatments: In the waiting room. Nature.2012; 491(7422):S14-S16.

9. FACHINETTI TA, et al. Processo de Construção de Recurso de Tecnologia Assistiva para Aluno com Paralisia Cerebral em Sala de Recursos Multifuncionais. Rev. bras. educ. espec. 2017;23(4):547-562.

10. FERREIRA NR, RANIERI LP. O uso da tecnologia assistiva por professores de educação física. Revista Eletrônica de Educação. 2016;10(3):215-229.

11. GONCALVES AP, et al. Transtornos do espectro do autismo e psicanálise: revisitando a literatura. Tempo psicanal. 2017 ; 49(2):152-181.

12. GRIESI-OLIVEIRA K, SERTIE, AL. Transtornos do espectro autista: um guia atualizado para aconselhamento genético. Einstein (São Paulo). 2017;15(2):233-238.

13. MECCA TP, et al. Rastreamento de sinais e sintomas de transtornos do espectro do autismo em irmãos. Rev. psiquiatr. 2011;33(2):116-120.

14. MENEZES CGL, PERISSINOTO, J. Habilidade de atenção compartilhada em sujeitos com transtornos do espectro autístico. Pró-Fono R. Atual. Cient.2008; 20(4):273-278.

15. MIELE FG, AMATO CAIH. Transtorno do espectro autista: qualidade de vida e estresse em cuidadores e/ou familiares revisão de literatura. Cad. Pós-Grad. Distúrb. Desenvolv. 2016;16(2): 89-102.

16. MONTEIRO AF, et al. Considerações sobre critérios diagnósticos de transtorno do espectro autista, e suas implicações no campo científico. Universidade de Caxias do Sul - DO CORPO: Ciências e Artes, 2017;7(1):87-97

17. MORESI EAD, et al. Tecnologia assistiva e autismo. Memorias de la Octava Conferência Iberoamericana de Complejidad, informática y cibernética (CICIC 2018). 2018.

18. PEREIRA ACS et al. Transtorno do Espectro Autista (TEA): definição, características e atendimento educacional. Educação Batatais. 2015; 5(2):191-212.

19. RABELLO S, et al. The influence of assistive technology devices on the performance of activities by visually impaired. Rev. bras.oftalmol. 2014; 73(2):103-105.

20. ROCHA ANDC, DELIBERATO D. Tecnologia assistiva para a criança com paralisia cerebral na escola: identificação das necessidades. Rev. bras. educ. espec. 2012; 18(1):71-92.

21. SANTOS RK. Transtorno do espectro do autismo (TEA): do reconhecimento à inclusão no âmbito educacional. Universidade Federal Rural do Semi-Árido. 2017; 3:219-232.

22. SCHWARTZMAN JS, et al. The eye-tracking of social stimuli in patients with Rett syndrome and autism spectrum disorders: a pilot study. Arq. Neuro-Psiquiatr. 2015;73(5):402-407.

23. SEABRA JUNIOR MO, LACERDA LCZ. Atendimento Educacional Especializado: planejamento e uso do recurso pedagógico.Rev. Bras. Educ. 2018;23(23):230-246.

24. TAMANAHA AC, et al. Trajetória de aquisição e desenvolvimento de fala de crianças autistas com e sem história de regressão autística. CoDAS. 2014;26(4):265-269.

25. TENÓRIO MCA, et al. Autismo: a tecnologia como ferramenta assistiva ao processo de ensino e aprendizagem de uma criança dentro do espectro. CINTEDI- Práticas pedagógicas direitos humanos e interculturalidade. 2015.

26. ZAUZA CMF, et al. O processo de inclusão de portadores do transtorno do espectro autista. In: Psicologia-portal da psicologia.2015; disponível em: http://www.psicologia.pt/artigos/textos/A0896.pdf. Acesso em: 24 de ago.de 2019.

27. CARVALHO FILHA FSS, et al. Entendimento do espectro autista por pais/cuidadores - estudo descritivo. Revista Científica Sena Aires. 2018; 7(2):105-116.

28. CARVALHO FILHA FSS, et al. Coping e estresse familiar e enfrentamento na perspectiva do transtorno do espectro do autismo. Revista Científica Sena Aires. 2018;7(1):23-30

29. CARVALHO-FILHA FSS, et al. O cuidado multiprofissional e familiar à pessoa no espectro do autismo: a importância da complementaridade terapêutica. In Nascimento FSC, et al., editores. 1 ${ }^{\text {a }}$. ed. Fortaleza: Imprece; 2019. p.233-252. 